\title{
Linear and nonlinear wave propagation in coated or uncoated elastic half-spaces
}

\author{
BY Y.B. Fu \\ Department of Mathematics, Keele University, Staffordshire St5 5BG, UK
}

In these lectures, we discuss the following three closely related topics.

(i) Unification of different methods for deriving evolution equations for surface acoustic waves. Early studies on nonlinear surface acoustic waves were thwarted by very complicated derivation of evolution equations. Worse still, different methods seemed to have given different evolution equations. Later on, it became known that all these methods except one yield the same evolution equation, but even at the time when we started to prepare the current lecture notes, there still existed a method that does not agree with the other methods. Such a situation is unsatisfatory since each method has some following. The purpose of the lectures on this topic is three-fold. Firstly, we aim to show that derviation of the evolution eqution for nonlinear surface waves can be carried out in one A4 page even in the most general case. Secondly, we show that the odd method that used to give a different evolution equation can in fact be used to obtain the same evolution equation if it is properly executed. Thus, we set the record straight: all known methods should and do give the same evolution equation! Thirdly, we express our evolution equation in terms of results from the linear surface-wave theory built on the Stroh formulation, and we explain how the coefficients in the evolution equation can be evaluated efficiently.

(ii) Linear wave propagation in a coated elastic half-space. This is partly in preparation for our discussion of the third topic, but the problem is of much interest in its own right. We show how the dispersion relation can be expressed elegantly in terms of the surface-impedance matrices associated with the layer and the half-space. We derive a two-term expression for the wave speed in the long-wavelength limit.

(iii) Periodic and solitary waves in a coated elastic half-space. An uncoated elastic half-space cannot in general support solitary waves due to lack of dispersion although it has been argued previously that the nonlocal character of nonlinearity may give rise to the existence of steady travelling waves. We derive the nonlinear evolution equation for small-amplitude long-wavelength travelling 
waves propagating in a coated elastic half-space where the thin coating induces weak dispersion. When this evolution equation is linearized, we recover the two-term dispersion relation obtained in (ii). We explain a simple method that can be used to compute periodic or solitary travelling-wave solutions.

\section{Keywords: Nonlinear surface waves; elastic half-space; coated half-space; Stroh formalism; nonlinear elasticity; prestress}

\section{Introduction - background and literature review}

Elastic surface waves are travelling waves that can propagate along the surface of an elastic half-space. They satisfy the traction-free boundary condition and decay to zero exponentially away from the surface. Understanding of nonlinear effects on the evolution of surface waves is known to have applications in signal processing, material characterization, and non-destructive evaluation; see, for instance, Parker and Maugin (1987), Mayer (1995), Maugin (1999), Hess (2002) and the references therein. More recently, it has also found applications in nanotechnology; see Hess and Lomonosov (2005), Hess et al. (2005). Historically, evolution equations for nonlinear acoustic surface waves have been derived using different procedures and it was very often not immediately clear whether one evolution equation was equivalent to another. It seems that derivation of evolution equations for nonlinear surface waves was actually initiated by Reutov (1973), but this paper remained unnoticed in the West for a considerable period of time. Many researchers thought that Kalyanasundaram's 1981 paper was the first where a multiple scale approach was used to study the evolution of nonlinear surface waves. In both of these two papers, a far-distance variable $X\left(=\varepsilon x_{1}\right)$ and a slow time variable $\tau(=\varepsilon t)$ were introduced, where $x_{1}$ is the coordinate along the direction of propagation, $t$ is time and $\varepsilon$ is a small parameter characterizing the amplitude of strains. When Kalyanasundaram's (1981) method (hereafter referred to as Method I) is used, secular terms appear in the $O\left(\varepsilon^{2}\right)$ solution. This 'deficiency' was later remedied by Lardner (1983) through the introduction of another far-distance variable $\eta\left(=\varepsilon x_{2}\right)$ where $x_{2}$ is the coordinate such that $x_{2}>0$ defines the half-space. We refer to this as Method II. This method was followed by Lardner (1984, 1985, 1986), Lardner and Tupholme (1986), David (1985), Harvey et al. (1992), Harvey and Tupholme (1991, 1992), Tupholme and Harvey $(1988,1992)$. The use of multiple scale $\eta$ was also independently proposed by Planat (1985) but he assumed that the dependence on $\eta$ and $X$ was only through a linear combination of $X$ and $\eta$ with unspecified coefficients. Method III is Parker et al.'s (1992) projection method which can immediately be recognized as being equivalent to Method I and the approaches used in Parker (1988) and Hunter (1989). Method IV is Reutov (1973)'s and Zabolotskaya's (1992) Hamiltonian formalism which was followed by Shull et al. (1993), Hamilton et al. (1995, 1999). Method V is Fu and 
Devenish's (1996) virtual-work method which incorporated the best features of the projection method and the Hamiltonian formalism. All the above methods, although seemingly different, work with the frequency domain and are based essentially on the same underlying philosophy. It was explicitly recognized in Fu and Devenish's (1996) and Eckl et al. (2004) that Methods I-IV yield the same evolution equation for the surface velocity/elevation although this was not immediately clear from the papers in which these methods were first presented. In Reutov (1973)'s pioneering paper, it was already recognized that the projection method was equivalent to the Hamiltonian formalism.

In contrast with the above methods, Method VI proposed by Gusev et al. (1997, 1998) works with the time domain and is recognized as being different from the other methods even if it is translated into the frequency domain; see Meegan et al. (1999) and Eckl et al. (2004). Method VI was followed by Kolomenskii et al. (1997), Kolomenskii and Schuessler (2001), Kolomenskii et al. (2003), Jerebtsov et al. (2004). It seems that Gusev et al. $(1997,1998)$ did not realize that the evolution equation for the surface velocity is independent of whether the far-distance variable $\eta=\varepsilon x_{2}$ is introduced or not and they advocated the importance of restoring this variable in deriving the evolution equation for the surface velocity. One of the motivations for the present study is to understand why Method VI gives a different evolution equation. We shall show that Method VI is in fact able to yield the same evolution equation as the other methods if a certain underlying assumption is removed. Thus, all the existing methods can now be said to be equivalent.

Another motivation for the present study is that when studying nonlinear surface waves in generally anisotropic elastic materials, previous investigators seem to have been oblivious of the more recent developments concerning linear surface waves. The many beautiful results concerning linear surface waves based on the Stroh formulation (Stroh 1958) should not only facilitate numerical evaluation of the surface-wave speed and coefficients in the evolution equation, but they also contain a lot of qualitative information about properties of linear surface waves. For instance, it could be misleading not to write the secular equation for the wave speed as a real relation, since otherwise new researchers could question whether it would be possible to find a real root (the wave speed) to satisfy a complex equation (which contains two real equations).

With the aid of the evolution equation derived, it can easily be shown that initially smooth profiles of surface displacement would evolve into shocks within a finite time. This is to be expected since travelling waves propagating in an uncoated elastic half-space are non-dispersive. A natural follow-up problem is the propagation of nonlinear travelling waves, and in particular solitary waves, in a coated elastic half-space where a thin coating gives rise to dispersion. Linear travelling waves in a 
coated elastic half-space were first studied by Tiersten (1969). Recently, Ogden and Sotiropoulos $(1995,1996)$ studied linear travelling waves in a pre-stressed, coated, incompressible or compressible, elastic half-space. When a prestress is present, there arises the possibility of existence of static sinusoidal solutions which has stability implications; see also Bigoni et al. (1997), Cai and Fu (1999, 2000) for further details and additional references. However, our attention here will be focussed on travelling wave solutions. We shall demonstrate that the surface-impedance matrix plays an important role in studying such waves and in terms of it the dispersion relation can be written in a very compact and revealing form.

Nonlinear travelling waves in a coated elastic half-space have previously been studied by Porubov and Samsonov (1995), Eckl and Mayer (1998), and Eckl et al. (2004). Porubov and Samsonov (1995) focussed on finding solitary wave solutions that have analytical/explicit expressions and showed that when the coating layer is perfectly bonded to the half-space (as we assume here), such solutions do not exist. Our treatment of the problem follows the spirit of the last two papers where the authors found solitary wave solutions numerically. In these two papers, the authors replaced the action of the coating layer by an effective boundary condition applied to the surface of the half-space. We show how this effective boundary condition can be derived and again how the evolution equation can be derived with the aid of the virtual work method. We explain a simple method that can be used to compute periodic and solitary travelling wave solutions.

\section{Unification of different methods for deriving evolution equations for surface acoustic waves}

\section{(a) Governing equations and linear theory}

We shall first consider a homogeneous, unstressed, generally anisotropic elastic half-space defined by

$$
0<x_{2}<\infty, \quad-\infty<x_{1}, x_{3}<\infty
$$

relative to a rectangular coordinate system with coordinates $\left(x_{i}\right)$. Free surface waves are governed by the equation of motion

$$
\sigma_{i j, j}=\rho \ddot{u}_{i}, \quad 0<x_{2}<\infty
$$

the traction-free boundary condition

$$
\sigma_{i 2}=0 \quad \text { on } x_{2}=0,
$$

and the decay condition

$$
u_{k} \rightarrow 0 \quad \text { as } x_{2} \rightarrow \infty
$$


where $\left(\sigma_{i j}\right)$ is the stress tensor, $\left(u_{k}\right)$ the displacement, $\rho$ the material density, a comma and a superimposed dot denote differentiation with respect to the spatial coordinates and time, respectively. The above equations are closed by the constitutive relation given by

$$
\sigma_{i j}=c_{i j k l} u_{k, l}+\frac{1}{2} e_{i j k l m n} u_{k, l} u_{m, n}+\cdots,
$$

where $c_{i j k s}$ and $e_{i j k l m n}$ are tensors of first- and second-order elastic moduli. We assume that $c_{i j k l}$ and $e_{i j k l m n}$ satisfy the pairwise symmetry relations $c_{i j k l}=c_{k l i j}$, $e_{i j k l m n}=e_{k l i j m n}=e_{i j m n k l}$, and that $c_{i j k l}$ satisfies the strong convexity condition, but otherwise the material is generally anisotropic. We observe the summation convention whereby all repeated suffices are summed from 1 to 3 . At the end of these notes, we shall explain how results obtained for a generally anisotropic material can be applied to a generally prestressed isotropic material.

We now consider the linearized form of (2.1)-(2.3) and look for a travelling-wave solution of the form

$$
\mathbf{u}=\mathbf{a} \mathrm{e}^{\mathrm{i} \omega p x_{2} / v} \cdot \mathrm{e}^{-\mathrm{i} \omega \theta}, \quad \theta=t-x_{1} / v
$$

where $\omega>0$ is the frequency, $v$ the speed and the constant $p$ and amplitude vector a are to be determined.

On substituting (2.5) into $c_{i j k l} u_{k, j l}=\rho \ddot{u}_{i}$, the linearized form of (2.1), we find that $p$ and $\mathbf{a}$ are determined by the eigenvalue problem

$$
\left(p^{2} T+p\left(R+R^{T}\right)+Q-\rho v^{2} I\right) \mathbf{a}=\mathbf{0},
$$

where $I$ is the identity matrix, the superscript " $T$ " denotes matrix transpose, and the components of the three matrices $T, R, Q$ are defined by

$$
T_{i k}=c_{i 2 k 2}, \quad R_{i k}=c_{i 1 k 2}, \quad Q_{i k}=c_{i 1 k 1} .
$$

Since $c_{i j k s}$ satisfies the strong convexity condition, the eigenvalues of $p$ in (2.6) cannot be pure real when $v=0$ and they will remain complex until $v=\hat{v}$ at which at least one pair of eigenvalues first become pure real. The $\hat{v}$ is usually referred to as the limiting speed (Chadwick and Smith 1977) and surface waves with $v<\hat{v}$ are said to be subsonic. An elegant result in anisotropic elasticity is that a unique free-surface wave should normally exist except in some special cases (Barnett and Lothe 1974).

To characterize the linear free-surface wave solution, we assume from now on that $v<\hat{v}$ and denote by $p^{(1)}, p^{(2)}, p^{(3)}$ the three eigenvalues of $p$ with positive imaginary parts and $\mathbf{a}^{(1)}, \mathbf{a}^{(2)}, \mathbf{a}^{(3)}$ the associated eigenvectors. Then a general solution that satisfies the decaying condition $(2.3)$ is

$$
\mathbf{u}=\left(\sum_{j=1}^{3} c_{j} \mathbf{a}^{(j)} \mathrm{e}^{\mathrm{i} \omega p^{(j)} x_{2} / v}\right) \mathrm{e}^{-\mathrm{i} \omega \theta}=A\left\langle\mathrm{e}^{\mathrm{i} \omega p x_{2} / v}\right\rangle \mathbf{c} \mathrm{e}^{-\mathrm{i} \omega \theta}
$$


6

where $c_{1}, c_{2}, c_{3}$ are constants,

$$
A=\left[\mathbf{a}^{(1)}, \mathbf{a}^{(2)}, \mathbf{a}^{(2)}\right], \quad \mathbf{c}=\left[c_{1}, c_{2}, c_{3}\right]^{T},
$$

and $\left\langle\mathrm{e}^{\mathrm{i} \omega p x_{2} / v}\right\rangle$ denotes the diagonal matrix

$$
\operatorname{diag}\left\{\mathrm{e}^{\mathrm{i} \omega p^{(1)} x_{2} / v}, \mathrm{e}^{\mathrm{i} \omega p^{(2)} x_{2} / v}, \mathrm{e}^{\mathrm{i} \omega p^{(3)} x_{2} / v}\right\}
$$

The linearized boundary condition $c_{i 2 k l} u_{k, l}=0$ can be written as

$$
R^{T} \mathbf{u}_{, 1}+T \mathbf{u}_{, 2}=0
$$

On substituting the general solution (2.8) into (2.9), we obtain $B \mathbf{c}=\mathbf{0}$, where

$$
B=\left[\mathbf{b}^{(1)}, \mathbf{b}^{(2)}, \mathbf{b}^{(2)}\right]=R^{T} A+T A\langle p\rangle, \quad \mathbf{b}^{(j)}=\left(R^{T}+p^{(j)} T\right) \mathbf{a}^{(j)}
$$

without summation over $j$, and $\langle p\rangle=\operatorname{diag}\left\{p_{1}, p_{2}, p_{3}\right\}$.

At this juncture, we introduce the surface-impedance matrix $M$ (Ingebrigtsen and Tonning 1969) through

$$
M=-\mathrm{i} B A^{-1}
$$

In terms of this matrix, the boundary condition $B \mathbf{c}=\mathbf{0}$ may be rewritten as

$$
M \mathbf{d}=\mathbf{0}, \quad \text { where } \mathbf{d}=A \mathbf{c}
$$

We remark that it is advantageous to use $M$ instead of $B$. Among its many useful properties, we mention that $M$ is Hermitian so that the secular equation $\operatorname{det} M=0$ for the surface-wave speed is real even for the most general anisotropic material (Stroh 1962). In the early studies on surface waves, it was not realized that the secular equation for the wave speed could always be written as a real equation, and as a result it was thought that existence of surface waves in anisotropic materials could only be exceptional (see, e.g., Farnell 1970). Also, all the eigenvalues of $M$ are monotone decreasing functions of $v$ (Barnett and Lothe 1985). As a result, $\operatorname{det} M=0$ will not have any spurious roots, which is a useful property when $\operatorname{det} M=0$ is solved numerically.

The surface-impedance matrix also has many applications other than in the surface-wave theory (see, e.g., Fu 2005). There now exist very efficient methods for computing this matrix. Firstly, this matrix has an integral representation given by

$$
M=\left(\int_{0}^{\pi} T_{\theta}^{-1} d \theta\right)^{-1}\left(\pi I-\mathrm{i} \int_{0}^{\pi} T_{\theta}^{-1} R_{\theta}^{\mathrm{T}} d \theta\right)
$$

where

$$
\begin{aligned}
T_{\theta} & =\cos ^{2} \theta T-\sin \theta \cos \theta\left(R+R^{\mathrm{T}}\right)+\sin ^{2} \theta\left(Q-\rho v^{2} I\right), \\
R_{\theta} & =\cos ^{2} \theta R-\sin ^{2} \theta R^{\mathrm{T}}+\sin \theta \cos \theta\left(T-Q+\rho v^{2} I\right), \\
Q_{\theta} & =\cos ^{2} \theta\left(Q-\rho v^{2} I\right)+\sin \theta \cos \theta\left(R+R^{\mathrm{T}}\right)+\sin ^{2} \theta T .
\end{aligned}
$$


This integral representation was first derived by Barnett and Lothe (1973), and later rederived by Mielke and Fu (2004) using a different procedure. Secondly, the surface-impedance matrix can also be computed with the aid of the matrix Riccati equation

$$
(M-\mathrm{i} R) T^{-1}\left(M+\mathrm{i} R^{\mathrm{T}}\right)-Q+\rho v^{2} I=0,
$$

see Biryukov (1985), Mielke and Sprenger (1998), Fu and Mielke (2002). Finally, when $x_{3}=0$ is a plane of material symmetry, this matrix has a simple and explicit expression ( Fu 2005, Fu and Brookes 2006).

A simple method for computing the surface-wave speed $v$ and the corresponding $M$ is as follows. Increase $v$ gradually from $v=0$ and at each step use (2.13) to evaluate $M$ and hence $\operatorname{det} M$. As soon as $\operatorname{det} M$ changes sign, use the corresponding values of $M$ and $v$ as a initial guess and solve (2.15) and $\operatorname{det} M=0$ to find $M$ and $v$ accurately.

In the following, we assume that $v$ has been determined as the unique solution of $\operatorname{det} M=0$ and $\mathbf{d}$ the corresponding non-trivial solution of $(2.12)_{1}$.

With the use of $(2.12)_{2}$, the solution (2.8) may be written as

$$
\mathbf{u}=\mathbf{u}\left(\theta, x_{2}, \omega\right)=A\left\langle\mathrm{e}^{\mathrm{i} \omega p x_{2} / v}\right\rangle A^{-1} \mathbf{d} \mathrm{e}^{-\mathrm{i} \omega \theta}
$$

We observe that this solution is only valid for $\omega>0$. When $\omega<0$, we would need to use $\bar{p}_{1}, \bar{p}_{2}, \bar{p}_{2}$ in the construction of the general decaying solution (2.8), where an overbar denotes complex conjugation. As a result, when $\omega<0$, we have

$$
\mathbf{u}\left(\theta, x_{2}, \omega\right)=\bar{A}\left\langle\mathrm{e}^{\mathrm{i} \omega \bar{p} x_{2} / v}\right\rangle \bar{A}^{-1} \overline{\mathbf{d}} \mathrm{e}^{-\mathrm{i} \omega \theta}
$$

We note that

$$
\mathbf{u}\left(\theta, x_{2}, \omega\right)=\overline{\mathbf{u}\left(\theta, x_{2},-\omega\right)}, \quad \text { for } \omega<0,
$$

and we remark that this rule of defining a frequency-dependent function when the frequency is negative in terms of the same function when the frequency is positive applies to all frequency-dependent functions in our subsequent analysis. To facilitate analysis later, we define a new function $\mathbf{z}$ through

$$
\mathbf{z}\left(\omega, x_{2}\right)=A\left\langle\mathrm{e}^{\mathrm{i} \omega p x_{2} / v}\right\rangle A^{-1} \mathbf{d}, \quad \text { when } \omega>0 .
$$

As remarked above, we have $\mathbf{z}\left(\omega, x_{2}\right)=\overline{\mathbf{z}\left(-\omega, x_{2}\right)}$ when $\omega<0$ is negative.

Once the linear solution is known, we may construct an asymptotic solution

$$
\mathbf{u}=\varepsilon \mathbf{u}^{(1)}+\varepsilon^{2} \mathbf{u}^{(2)}+O\left(\varepsilon^{3}\right)
$$

for the original nonlinear surface-wave problem with

$$
\mathbf{u}^{(1)}=\frac{1}{2 \pi} \int_{-\infty}^{\infty} \tilde{\mathbf{u}}^{(1)} \mathrm{e}^{-\mathrm{i} \omega \theta} d \omega
$$


8

$$
\tilde{\mathbf{u}}^{(1)}=\mathcal{F}\left[\mathbf{u}^{(1)}\right] \equiv \int_{-\infty}^{\infty} \mathbf{u}^{(1)} \mathrm{e}^{\mathrm{i} \omega \theta} d \theta=f(\omega, \tau, \eta) \mathbf{z}\left(\omega, x_{2}\right)
$$

where the unknown amplitude function $f(\omega, \tau, \eta)$ is assumed to depend on the slowtime variable $\tau=\varepsilon t$ and the far-distance variable $\eta=\varepsilon x_{2}$, and to satisfy the condition

$$
f(-\omega, \tau, \eta)=\overline{f(\omega, \tau, \eta)}
$$

However, we shall show later that the evolution equation for the surface velocity is independent of whether the far-distance variable $\eta$ is introduced or not. Throughout this paper we use both $\mathcal{F}[g]$ and $\tilde{g}$ to denote the Fourier transform of a function $g$.

The remaining of this subsection is devoted to explaining why the method used by Gusev et al. (1998) yields a different evolution from the other methods. If you simply want to learn the easiest method for deriving the evolution equation for surface acoustic waves, you may skip the rest of this subsection and the next subsection, and go straight to the subsection entitled Evolution equation using the virtual work method.

With the aid of (2.19) and (2.22), we deduce that

$$
\frac{\partial \tilde{\mathbf{u}}^{(1)}}{\partial x_{2}}=f(\omega, \tau, \eta) \frac{\partial \mathbf{z}}{\partial x_{2}}=\frac{\mathrm{i} \omega}{v} \cdot \begin{cases}A\langle p\rangle A^{-1} \tilde{\mathbf{u}}^{(1)}, & \text { when } \omega>0 \\ \bar{A}\langle\bar{p}\rangle \bar{A}^{-1} \tilde{\mathbf{u}}^{(1)}, & \text { when } \omega<0 .\end{cases}
$$

Thus, following Gusev et al. (1998) we have

$$
\frac{\partial \tilde{\mathbf{u}}^{(1)}}{\partial x_{2}}=-\frac{1}{v}(\operatorname{Re} G+\mathrm{i} \operatorname{sgn}(\omega) \operatorname{Im} G) \mathcal{F}\left[\frac{\partial \mathbf{u}^{(1)}}{\partial \theta}\right],
$$

where

$$
G=A\langle p\rangle A^{-1},
$$

Re and Im denote the real and imaginary parts, respectively, and sgn is the sign function. The matrix $G$ corresponds to the matrix $\beta$ defined in Gusev et al. (1998). It follows from $(2.10),(2.11)$ and the Hermitian property $M^{T}=\bar{M}$ that

$$
G^{T} T=\mathrm{i} M^{T}-R, \quad T \bar{G}=-\mathrm{i} M^{T}-R^{T},
$$

which will be used in our derivations later. We also observe that $G$ is related to the matrix $E$ of $\mathrm{Fu}$ and Mielke (2002) by

$$
G=\mathrm{i} E \text {. }
$$

The matrix $E$ plays an important role in the linear surface-wave theory. It can be shown that

$$
E=T^{-1}\left(M+\mathrm{i} R^{T}\right), \quad E \mathbf{a}^{(k)}=\mathrm{i} p^{(k)} \mathbf{a}^{(k)}, \quad k=1,2,3,
$$


the second relation showing that the three eigenvalues of $E$ are $\mathrm{i} p_{1}, \mathrm{i} p_{2}, \mathrm{i} p_{3}$. These relations can be used to compute $\mathbf{a}^{(k)}$ and $p^{(k)}$ more efficiently than (2.6) once the surface-impedance matrix $M$ is known (e.g. from (2.13)).

Taking the inverse Fourier transform of (2.23), we have

$$
\frac{\partial \mathbf{u}^{(1)}}{\partial x_{2}}=-\frac{1}{v}\left\{\operatorname{Re} G \frac{\partial \mathbf{u}^{(1)}}{\partial \theta}-(\operatorname{Im} G) H\left[\frac{\partial \mathbf{u}^{(1)}}{\partial \theta}\right]\right\},
$$

where $H$ denotes the Hilbert transform defined by

$$
H[g(\theta)]=\frac{1}{\pi} \mathrm{p} . \mathrm{v} \cdot \int_{-\infty}^{\infty} \frac{g(y)}{y-\theta} d y=-\frac{1}{\pi \theta} \star g(\theta),
$$

and use has been made of the basic result that

$$
\mathcal{F}\left[\text { p.v. } \frac{1}{\theta}\right]=\text { p.v. } \int_{-\infty}^{\infty} \frac{1}{\theta} \mathrm{e}^{\mathrm{i} \omega \theta} d \theta=2 \mathrm{i} \lim _{a \rightarrow 0} \int_{a}^{\infty} \frac{\sin \omega \theta}{\theta} d \theta=\mathrm{i} \pi \operatorname{sgn}(\omega) .
$$

In (2.29) and (2.30), p.v. denotes "principal value" and the star denotes integral convolution.

On differentiating (2.20) with respect to $x_{2}$ and making use of $(2.28)$, we obtain

$$
\begin{aligned}
\frac{\partial \mathbf{u}}{\partial x_{2}} & =-\frac{\varepsilon}{v}\left\{\operatorname{Re} G \frac{\partial \mathbf{u}^{(1)}}{\partial \theta}-(\operatorname{Im} G) H\left[\frac{\partial \mathbf{u}^{(1)}}{\partial \theta}\right]\right\}+O\left(\varepsilon^{2}\right), \\
& =-\frac{1}{v}\left\{\operatorname{Re} G \frac{\partial \mathbf{u}}{\partial \theta}-(\operatorname{Im} G) H\left[\frac{\partial \mathbf{u}}{\partial \theta}\right]\right\}+O\left(\varepsilon^{2}\right) .
\end{aligned}
$$

It can further be deduced with the aid of the property $H[H[g(x)]]=-g(x)$ that

$$
\frac{\partial^{2} \mathbf{u}}{\partial x_{2}^{2}}=\frac{1}{v^{2}}\left\{\operatorname{Re}\left(G^{2}\right) \frac{\partial^{2} \mathbf{u}}{\partial \theta^{2}}-\operatorname{Im}\left(G^{2}\right) H\left[\frac{\partial^{2} \mathbf{u}}{\partial \theta^{2}}\right]\right\}+O\left(\varepsilon^{2}\right) .
$$

Gusev et al. (1998) made a fundamental assumption that effectively says that the $O\left(\varepsilon^{2}\right)$ terms in (2.31) and (2.32) are identically zero (see their statement in the paragraph between equations (29) and (30)). Thus, a major operation used by Gusev et al. (1998) is to use (2.31) and (2.32), with the $O\left(\varepsilon^{2}\right)$ terms neglected, to eliminate derivatives with respect to $x_{2}$ in favor of derivatives with respect to $\theta$. When this operation is applied to quadratic terms, it will induce an error of order $\varepsilon^{3}$ which can be neglected in the derivation of the nonlinear evolution equation. However, they also applied this operation to linear terms and in doing so they neglected some $O\left(\varepsilon^{2}\right)$ terms which, we believe, are not identically zero. Thus, the derivation procedure used in Gusev et al. (1998) is asymptotically inconsistent. We believe that this is why their method would gave a different evolution equation from all the other methods. This is elaborated further in the following subsection. 
10

(b) Nonlinear evolution equation

With the variable transformation

$$
\left(x_{1}, x_{2}, t\right) \rightarrow\left(\theta, x_{2}, \tau, \eta\right)
$$

we have

$$
\frac{\partial}{\partial x_{1}} \rightarrow-\frac{1}{v} \frac{\partial}{\partial \theta}, \quad \frac{\partial}{\partial t} \rightarrow \frac{\partial}{\partial t}+\varepsilon \frac{\partial}{\partial \tau}, \quad \frac{\partial}{\partial x_{2}} \rightarrow \frac{\partial}{\partial x_{2}}+\varepsilon \frac{\partial}{\partial \eta}
$$

The nonlinear equation of motion (2.1) and the boundary condition (2.2) then become

$$
\begin{gathered}
\left(\rho I-\frac{1}{v^{2}} Q\right) \frac{\partial^{2} \mathbf{u}}{\partial \theta^{2}}+\frac{1}{v}\left(R+R^{T}\right) \frac{\partial^{2} \mathbf{u}}{\partial \theta \partial x_{2}}-T \frac{\partial^{2} \mathbf{u}}{\partial x_{2}^{2}}=-2 \rho \varepsilon \frac{\partial^{2} \mathbf{u}}{\partial \theta \partial \tau}+2 \varepsilon T \frac{\partial^{2} \mathbf{u}}{\partial x_{2} \partial \eta} \\
-\frac{1}{v}\left(R+R^{T}\right) \varepsilon \frac{\partial^{2} \mathbf{u}}{\partial \theta \partial \eta}-\frac{1}{v} \frac{\partial \mathbf{n}^{(1)}}{\partial \theta}+\frac{\partial \mathbf{n}^{(2)}}{\partial x_{2}}+O\left(\varepsilon^{3}\right), \quad 0<x_{2}<\infty \\
-\frac{1}{v} R^{T} \frac{\partial \mathbf{u}}{\partial \theta}+T \frac{\partial \mathbf{u}}{\partial x_{2}}=-\varepsilon T \frac{\partial \mathbf{u}}{\partial \eta}-\mathbf{n}^{(2)}+O\left(\varepsilon^{3}\right), \quad x_{2}=\eta=0
\end{gathered}
$$

where the two vector functions $\mathbf{n}^{(1)}, \mathbf{n}^{(2)}$ are defined by

$$
n_{i}^{(1)}=\frac{1}{2} e_{i 1 k l m n} u_{k, l} u_{m, n}, \quad n_{i}^{(2)}=\frac{1}{2} e_{i 2 k l m n} u_{k, l} u_{m, n} .
$$

At this stage, Gusev et al. (1998) would inconsistently apply the substitutions (2.31) and (2.32) to the linear terms of both order $\varepsilon$ and order $\varepsilon^{2}$ in (2.33) and (2.34) and to obtain

$$
\begin{gathered}
2 \rho \varepsilon \frac{\partial^{2} \mathbf{u}}{\partial \theta \partial \tau}+\frac{1}{v}\left(R+R^{T}\right) \varepsilon \frac{\partial^{2} \mathbf{u}}{\partial \theta \partial \eta}+\frac{2}{v} \varepsilon T\left\{\operatorname{Re} G \frac{\partial^{2} \mathbf{u}}{\partial \theta \partial \eta}-(\operatorname{Im} G) H\left[\frac{\partial^{2} \mathbf{u}}{\partial \theta \partial \eta}\right]\right\} \\
=-\frac{1}{v} \frac{\partial \mathbf{n}^{(1)}}{\partial \theta}+\frac{\partial \mathbf{n}^{(2)}}{\partial x_{2}}+O\left(\varepsilon^{3}\right), \quad 0<x_{2}<\infty \\
\frac{1}{v}\left\{R^{T}+T \operatorname{Re} G \frac{\partial \mathbf{u}}{\partial \theta}-T(\operatorname{Im} G) H\left[\frac{\partial \mathbf{u}}{\partial \theta}\right]\right\}=\varepsilon T \frac{\partial \mathbf{u}}{\partial \eta}+\mathbf{n}^{(2)}+O\left(\varepsilon^{3}\right), \quad x_{2}=\eta=0
\end{gathered}
$$

where use has been made of the fact that

$$
\rho v^{2} I-Q=\left(R+R^{T}\right) \operatorname{Re} G+T \operatorname{Re}\left(G^{2}\right), \quad 0=\left(R+R^{T}\right) \operatorname{Im} G+T \operatorname{Im}\left(G^{2}\right),
$$

which can be deduced from (2.6) with aid of (2.10) and (2.24).

Applying the Fourier transform to (2.36) and (2.37), we would obtain for $\omega>0$

$$
\begin{gathered}
\varepsilon\left\{2 \rho \frac{\partial \tilde{\mathbf{v}}}{\partial \tau}+\frac{1}{v}\left(R+R^{T}+2 T G\right) \frac{\partial \tilde{\mathbf{v}}}{\partial \eta}\right\}=\frac{1}{v} \mathrm{i} \omega \tilde{\mathbf{n}}^{(1)}+\frac{\partial \tilde{\mathbf{n}}^{(2)}}{\partial x_{2}}+O\left(\varepsilon^{3}\right), \quad 0<x_{2}<\infty \\
\frac{1}{v} \mathrm{i} M \tilde{\mathbf{v}}=\varepsilon T \frac{\partial \tilde{\mathbf{u}}}{\partial \eta}+\tilde{\mathbf{n}}^{(2)}+O\left(\varepsilon^{3}\right), \quad x_{2}=\eta=0
\end{gathered}
$$


where $\mathbf{v}=\partial \mathbf{u} / \partial t=\partial \mathbf{u} / \partial \theta$ and $\tilde{\mathbf{v}}$ denotes the Fourier transform of $\mathbf{v}$ so that

$$
\tilde{\mathbf{v}}=-\mathrm{i} \omega \tilde{\mathbf{u}}=-\varepsilon \mathrm{i} \omega f(\omega, \tau, \eta) \mathbf{z}\left(\omega, x_{2}\right)+O\left(\varepsilon^{2}\right) .
$$

Contracting (2.40) with the left eigenvector $\boldsymbol{\zeta}$ of $M$ and replacing $\tilde{\mathbf{u}}$ by $\tilde{\mathbf{v}} /(-\mathrm{i} \omega)$, we would eliminate the $O(\varepsilon)$ term on the left and obtain

$$
\varepsilon \boldsymbol{\zeta} \cdot T \frac{\partial \tilde{\mathbf{v}}}{\partial \eta}-\mathrm{i} \omega \boldsymbol{\zeta} \cdot \tilde{\mathbf{n}}^{(2)}+O\left(\varepsilon^{3}\right)=0
$$

It follows from $(2.25)$ that

$$
R+R^{T}+2 T G=2 \mathrm{i} M+R-R^{T} .
$$

Thus, (2.39) may also be written as

$$
\frac{\varepsilon}{v}\left(2 \mathrm{i} M+R-R^{T}\right) \frac{\partial \tilde{\mathbf{v}}}{\partial \eta}=-\varepsilon 2 \rho \frac{\partial \tilde{\mathbf{v}}}{\partial \tau}+\frac{1}{v} \mathrm{i} \omega \tilde{\mathbf{n}}^{(1)}+\frac{\partial \tilde{\mathbf{n}}^{(2)}}{\partial x_{2}}+O\left(\varepsilon^{3}\right), \quad 0<x_{2}<\infty .
$$

The evolution equation of Gusev et al. (1998) (in the frequency domain) would be obtained by taking the limit $x_{2} \rightarrow 0$ in (2.43), solving the resulting equation for $\partial \tilde{\mathbf{v}} / \partial \eta$ and substituting it into the boundary condition (2.42), the derivatives with respect to $x_{2}$ being eliminated with the aid of (2.31). However, this operation is illegitimate since $\partial \tilde{\mathbf{v}} / \partial \eta$ becomes parallel to $\mathbf{d}$ as $x_{2} \rightarrow 0$ so that

$$
\left.\left(2 \mathrm{i} M+R-R^{T}\right) \frac{\partial \tilde{\mathbf{v}}}{\partial \eta}\right|_{x_{2} \rightarrow 0}=\left.\left(R-R^{T}\right) \frac{\partial \tilde{\mathbf{v}}}{\partial \eta}\right|_{x_{2} \rightarrow 0},
$$

but the coefficient matrix $R-R^{T}$ is in general not invertible (an example is when the material is isotropic). Gusev et al. (1998) did not seem to realize this fact and wrote down (in our notation)

$$
\left.\frac{\partial \tilde{\mathbf{v}}}{\partial \eta}\right|_{x_{2} \rightarrow 0}=\frac{v}{\varepsilon}\left(2 \mathrm{i} M+R-R^{T}\right)^{-1} \times \operatorname{RHS} \text { of }\left.(2.43)\right|_{x_{2} \rightarrow 0} .
$$

Clearly this expression cannot be expected to be consistent with (2.41) since its right hand will not be parallel to $\mathbf{d}$.

We now return to (2.33) and (2.34) in order to derive the correction evolution equation. On substituting (2.20) into these equations and equating the coefficient of $\varepsilon^{2}$, we obtain

$$
\begin{aligned}
\left(\rho I-\frac{1}{v^{2}} Q\right) & \frac{\partial^{2} \mathbf{u}^{(2)}}{\partial \theta^{2}}+\frac{1}{v}\left(R+R^{T}\right) \frac{\partial^{2} \mathbf{u}^{(2)}}{\partial \theta \partial x_{2}}-T \frac{\partial^{2} \mathbf{u}^{(2)}}{\partial x_{2}^{2}}=-2 \rho \frac{\partial^{2} \mathbf{u}^{(1)}}{\partial \theta \partial \tau}+2 T \frac{\partial^{2} \mathbf{u}^{(1)}}{\partial x_{2} \partial \eta} \\
& -\frac{1}{v}\left(R+R^{T}\right) \frac{\partial^{2} \mathbf{u}^{(1)}}{\partial \theta \partial \eta}-\frac{1}{v} \frac{\partial \mathbf{n}^{(1)}}{\partial \theta}+\frac{\partial \mathbf{n}^{(2)}}{\partial x_{2}}, \quad 0<x_{2}<\infty \\
& -\frac{1}{v} R^{T} \frac{\partial \mathbf{u}^{(2)}}{\partial \theta}+T \frac{\partial \mathbf{u}^{(2)}}{\partial x_{2}}=-T \frac{\partial \mathbf{u}^{(1)}}{\partial \eta}-\mathbf{n}^{(2)}, \quad x_{2}=\eta=0
\end{aligned}
$$


12

where without introducing extra notation $\mathbf{n}^{(1)}$ and $\mathbf{n}^{(2)}$ are still given by (2.35) but with $\mathbf{u}$ on the right hand sides now replaced by $\mathbf{u}^{(1)}$.

Applying the Fourier transform to (2.44) and (2.45), we obtain

$$
\begin{gathered}
\left(\rho I-\frac{1}{v^{2}} Q\right) \omega^{2} \tilde{\mathbf{u}}^{(2)}+\frac{\mathrm{i} \omega}{v}\left(R+R^{T}\right) \frac{\partial \tilde{\mathbf{u}}^{(2)}}{\partial x_{2}}+T \frac{\partial^{2} \tilde{\mathbf{u}}^{(2)}}{\partial x_{2}^{2}}=-2 \rho \mathrm{i} \omega \frac{\partial \tilde{\mathbf{u}}^{(1)}}{\partial \tau}-2 T \frac{\partial^{2} \tilde{\mathbf{u}}^{(1)}}{\partial x_{2} \partial \eta} \\
-\frac{\mathrm{i} \omega}{v}\left(R+R^{T}\right) \frac{\partial \tilde{\mathbf{u}}^{(1)}}{\partial \eta}-\frac{\mathrm{i} \omega}{v} \tilde{\mathbf{n}}^{(1)}-\frac{\partial \tilde{\mathbf{n}}^{(2)}}{\partial x_{2}}, \quad 0<x_{2}<\infty \\
\frac{\mathrm{i} \omega}{v} R^{T} \tilde{\mathbf{u}}^{(2)}+T \frac{\partial \tilde{\mathbf{u}}^{(2)}}{\partial x_{2}}=-T \frac{\partial \tilde{\mathbf{u}}^{(1)}}{\partial \eta}-\tilde{\mathbf{n}}^{(2)}, \quad x_{2}=\eta=0
\end{gathered}
$$

We emphasize that in arriving at the equations (2.46) and (2.47) we have used the same procedure as Gusev et al. (1998) except that we have not assumed that $\partial \mathbf{u} / \partial x_{2}$ is related to $\partial \mathbf{u} / \partial \theta$ by exactly the same formula as $\partial \mathbf{u}^{(1)} / \partial x_{2}$ is related to $\partial \mathbf{u}^{(1)} / \partial \theta$. The latter assumption would force the left hand side of (2.46) to be identically zero and the left hand side of (2.47) to be orthogonal to the left eigenvector $\boldsymbol{\zeta}$ of $M$.

At this stage, the formulation can be connected to the formulations in Methods I-V. Any of these methods would yield the right evolution equation. Since the governing equation and the boundary condition are already expanded out, the best method for deriving the evolution equation from this point is probably the projection method (Parker et al. 1992). In the next subsection, however, we shall show that when the virtual-work method is used, there is in fact no need to expand the boundary condition.

In the project method we contract (2.46) with the linear solution $\mathbf{z}\left(-\omega, x_{2}\right)$ defined by (2.19). We write $\mathbf{z}\left(-\omega, x_{2}\right)$ as $\mathbf{z}^{-}$in order to avoid confusion with $\mathbf{z}\left(\omega, x_{2}\right)$ when the arguments are not written out. It can be shown by integrating by parts that

$$
\begin{array}{r}
\int_{0}^{\infty} \mathbf{z}^{-} \cdot \operatorname{LHS} \text { of }(2.46) d x_{2}=-\left.\mathbf{z}^{-} \cdot\left\{\frac{\mathrm{i} \omega}{v} R^{T} \tilde{\mathbf{u}}^{(2)}+T \frac{\partial \tilde{\mathbf{u}}^{(2)}}{\partial x_{2}}\right\}\right|_{x_{2}=0} \\
=\left.\mathbf{z}^{-} \cdot\left\{T \frac{\partial \tilde{\mathbf{u}}^{(1)}}{\partial \eta}+\tilde{\mathbf{n}}^{(2)}\right\}\right|_{x_{2}=0}=-\int_{0}^{\infty} \frac{\partial}{\partial x_{2}} \mathbf{z}^{-} \cdot\left\{T \frac{\partial \tilde{\mathbf{u}}^{(1)}}{\partial \eta}+\tilde{\mathbf{n}}^{(2)}\right\} d x_{2},
\end{array}
$$

where in obtaining the second equation above use has been made of (2.47). On replacing the left hand side (LHS) of (2.46) by its right hand side in (2.48), we obtain

$$
\begin{gathered}
\int_{0}^{\infty}\left\{-2 \rho \mathrm{i} \omega \mathbf{z}^{-} \cdot \frac{\partial \tilde{\mathbf{u}}^{(1)}}{\partial \tau}-T \mathbf{z}^{-} \cdot \frac{\partial^{2} \tilde{\mathbf{u}}^{(1)}}{\partial x_{2} \partial \eta}-\frac{\mathrm{i} \omega}{v} \mathbf{z}^{-} \cdot\left(R+R^{T}\right) \frac{\partial \tilde{\mathbf{u}}^{(1)}}{\partial \eta}\right. \\
\left.-\frac{\mathrm{i} \omega}{v} \mathbf{z}^{-} \cdot \mathbf{n}^{(1)}+\frac{\partial \mathbf{z}^{-}}{\partial x_{2}} \cdot \mathbf{n}^{(2)}+\frac{\partial \mathbf{z}^{-}}{\partial x_{2}} \cdot T \frac{\partial \tilde{\mathbf{u}}^{(1)}}{\partial \eta}\right\} d x_{2}=0 .
\end{gathered}
$$

We assume in the rest of this subsection that $\omega>0$. Then from (2.23),

$$
\frac{\partial \tilde{\mathbf{u}}^{(1)}}{\partial x_{2}}=\frac{\mathrm{i} \omega}{v} G \tilde{\mathbf{u}}^{(1)} .
$$


Similarly, we have

$$
\frac{\partial \mathbf{z}^{-}}{\partial x_{2}}=-\frac{i \omega}{v} \bar{G} \mathbf{z}^{-}
$$

With the aid of (2.50) and (2.51), the partial derivatives with respect to $x_{2}$ in the second and sixth terms in (2.49) can be eliminated. As a result, we obtain, after simplifying with the use of (2.25),

$$
\int_{0}^{\infty}\left\{-2 \rho \mathrm{i} \omega \mathbf{z}^{-} \cdot \frac{\partial \tilde{\mathbf{u}}^{(1)}}{\partial \tau}-\frac{\mathrm{i} \omega}{v} \mathbf{z}^{-} \cdot \tilde{\mathbf{n}}^{(1)}+\frac{\partial \mathbf{z}^{-}}{\partial x_{2}} \cdot \tilde{\mathbf{n}}^{(2)}\right\} d x_{2}=0
$$

or equivalently,

$$
\int_{0}^{\infty}\left\{-2 \rho \mathrm{i} \omega \mathbf{z}^{-} \cdot \frac{\partial \tilde{\mathbf{u}}^{(1)}}{\partial \tau}+\frac{1}{2} e_{i j k l m n} \mathcal{F}\left[z_{i, j}^{-}\right] \mathcal{F}\left[u_{k, l}^{(1)} u_{m, n}^{(1)}\right]\right\} d x_{2}=0 .
$$

It is seen that $\partial \tilde{\mathbf{u}}^{(1)} / \partial \eta$ has dropped out of the evolution equation (2.52) so that the evolution equation obtained from $(2.52)$ for $f(\omega, \tau, 0)$ would be independent of whether the far-distance variable $\eta$ has been introduced or not. The latter fact is of course already known (Fu and Devenish 1997, Eckl et al. 2004).

To obtain the final evolution equation from (2.52), we first obtain from (2.22)

$$
\begin{gathered}
\mathcal{F}\left[\frac{\partial \mathbf{u}^{(1)}}{\partial x_{1}}\right]=\frac{\mathrm{i} \omega}{v} \tilde{\mathbf{u}}^{(1)}=\frac{\mathrm{i} \omega}{v} f(\omega, \tau, \eta) A\left\langle\mathrm{e}^{\mathrm{i} \omega p x_{2} / v}\right\rangle \mathbf{c}, \\
\mathcal{F}\left[\frac{\partial \mathbf{u}^{(1)}}{\partial x_{2}}\right]=\frac{\mathrm{i} \omega}{v} f(\omega, \tau, \eta) A\left\langle p \mathrm{e}^{\mathrm{i} \omega p x_{2} / v}\right\rangle \mathbf{c} .
\end{gathered}
$$

Thus, we may write

$$
\mathcal{F}\left[u_{m, n}^{(1)}\right]=\frac{\mathrm{i} \omega}{v} f(\omega, \tau, \eta) Q_{m n}\left(x_{2}, \omega\right)
$$

where

$$
Q_{m n}\left(x_{2}, \omega\right)=\sum_{j=1}^{3} A_{m j} L_{n}^{(j)} \mathrm{e}^{\mathrm{i} \omega p^{(j)} x_{2} / v} c_{j}, \quad L_{n}^{(j)}=\delta_{n 1}+\delta_{n 2} p^{(j)}, \quad n, j=1,2,3,
$$

and we have written out the summation over $j$ explicitly since it is not a standard summation over a suffix repeated only once.

In terms of $Q_{m n}$ defined above, we have

$$
z_{i, j}^{-}=-\frac{\mathrm{i} \omega}{v} \overline{Q_{i j}\left(x_{2}, \omega\right)}
$$

and

$$
\begin{gathered}
\frac{1}{2} e_{i j k l m n} \mathcal{F}\left[u_{k, l}^{(1)} u_{m, n}^{(1)}\right]=\frac{1}{2} e_{i j k l m n} \mathcal{F}\left[u_{k, l}^{(1)}\right] \star \mathcal{F}\left[u_{m, n}^{(1)}\right] \\
=-\frac{1}{2 v^{2}} e_{i j k l m n} \int_{-\infty}^{\infty} \omega^{\prime}\left(\omega-\omega^{\prime}\right) f\left(\omega^{\prime}, \tau\right) f\left(\omega-\omega^{\prime}, \tau\right) Q_{k l}\left(x_{2}, \omega^{\prime}\right) Q_{m n}\left(x_{2}, \omega-\omega^{\prime}\right) d \omega^{\prime} .
\end{gathered}
$$


14

We note that when $\omega^{\prime}$ and $\omega-\omega^{\prime}$ are negative, the functions $Q_{k l}\left(x_{2}, \omega^{\prime}\right)$ and $Q_{m n}\left(x_{2}, \omega-\right.$ $\left.\omega^{\prime}\right)$ above are defined by the rule

$$
Q_{k l}\left(x_{2}, \omega^{\prime}\right)=\overline{Q_{k l}\left(x_{2},-\omega^{\prime}\right)}, \quad Q_{m n}\left(x_{2}, \omega-\omega^{\prime}\right)=\overline{Q_{m n}\left(x_{2}, \omega^{\prime}-\omega\right)},
$$

as prescribed below (2.18).

On substituting (2.53), (2.55) and (2.56) into (2.52), we obtain

$$
\frac{\partial f}{\partial \tau}=\frac{\mathrm{i}}{4 \rho v^{2} N(\omega)} \int_{-\infty}^{\infty} \omega^{\prime}\left(\omega-\omega^{\prime}\right) f\left(\omega^{\prime}\right) f\left(\omega-\omega^{\prime}\right) \mathcal{K}\left(\omega, \omega^{\prime}\right) d \omega^{\prime}
$$

where

$$
\begin{gathered}
N(\omega)=\int_{0}^{\infty} \boldsymbol{z} \cdot \overline{\boldsymbol{z}} d x_{2}=\int_{0}^{\infty} \mathbf{d} \cdot A^{-T}\left\langle\mathrm{e}^{\mathrm{i} \omega p x_{2} / v}\right\rangle A^{T} \bar{A}\left\langle\mathrm{e}^{-\mathrm{i} \omega \bar{p} x_{2} / v}\right\rangle \bar{A}^{-1} \overline{\mathbf{d}} d x_{2}=\frac{1}{\omega} N(1), \\
\mathcal{K}\left(\omega, \omega^{\prime}\right)=-\frac{\mathrm{i}}{v} e_{s j k l m n} \int_{0}^{\infty} Q_{k l}\left(x_{2}, \omega^{\prime}\right) Q_{m n}\left(x_{2}, \omega-\omega^{\prime}\right) \overline{Q_{s j}\left(x_{2}, \omega\right)} d x_{2}
\end{gathered}
$$

We note that the pair-wise symmetry of $e_{s j k l m n}$ implies that

$$
\mathcal{K}\left(\omega, \omega^{\prime}\right)=\mathcal{K}\left(\omega, \omega-\omega^{\prime}\right), \quad \mathcal{K}\left(\omega^{\prime}, \omega\right)=-\overline{\mathcal{K}\left(\omega, \omega^{\prime}\right)}
$$

\section{(c) Evolution equation using the virtual work method}

In this subsection we show how with the use of the virtual work method the same evolution can be obtained in the simplest manner. We assume throughout this subsection that $\omega_{0}$ is a positive constant and we consider a nonlinear surface-wave solution that is periodic in $\theta$ with period $2 \pi / \omega_{0}$. Such a solution can be represented as

$$
\mathbf{u}=\varepsilon \sum_{m=1}^{\infty} f_{m}(\tau) A\left\langle\mathrm{e}^{\mathrm{i} m \omega_{0} p x_{2} / v}\right\rangle A^{-1} \mathbf{d} \mathrm{e}^{-\mathrm{i} m \omega_{0} \theta}+C . C .+O\left(\varepsilon^{2}\right),
$$

where C.C. denotes the complex conjugate of the preceding term and $f_{m}(\tau)$ are amplitude functions to be determined. We have not allowed $f_{m}(\tau)$ to depend on the far-distance variable $\eta=\varepsilon x_{2}$ since the evolution equation for the surface elevation or surface velocity is independent of its inclusion.

The virtual work method starts with the following line integral:

$$
I=\lim _{h \rightarrow \infty} \oint_{C} \sigma_{i j} n_{j} \hat{u}_{i} d s
$$

where $\left(n_{i}\right)$ is the outward normal to the path, $\hat{\mathbf{u}}$ is a linear solution given by

$$
\hat{\mathbf{u}}=\mathbf{z}\left(-k \omega_{0}, x_{2}\right) \mathrm{e}^{\mathrm{i} k \omega_{0} \theta}=\overline{\mathbf{z}\left(k \omega_{0}, x_{2}\right)} \mathrm{e}^{\mathrm{i} k \omega_{0} \theta}, \quad k>0 \text { an integer }
$$

the closed path $C$ is the boundary of the rectangular region $\mathrm{S}$ : $\left[0 \leq \theta<2 \pi / \omega_{0}, 0 \leq\right.$ $\left.x_{2} \leq h\right]$, and $h$ is a positive constant. Since the integrand in (2.62) vanishes both on $x_{2}=0$ and as $x_{2} \rightarrow \infty$ and since it takes the same value on the two vertical paths 
due to periodicity, it is easy to see that $I$ is identically zero. With the use of the divergence theorem and the equation of motion (2.1), we also have

$$
I=\int_{0}^{\infty} d x_{2} \int_{0}^{2 \pi / \omega_{0}}\left\{\rho \hat{u}_{i} \frac{\partial^{2} u_{i}}{\partial \theta^{2}}+c_{i j k l} u_{k, l} \hat{u}_{i, j}+2 \rho \varepsilon \frac{\partial^{2} u_{i}}{\partial \theta \partial \tau} \cdot \hat{u}_{i}+\frac{1}{2} e_{i j k l m n} u_{k, l} u_{m, n} \hat{u}_{i, j}\right\} d \theta .
$$

Integrating the first two terms by parts and making use of the fact that $\mathbf{z}$ is a linear solution, we can show that the integral of the first two terms vanishes and (2.64) reduces to

$$
I=\int_{0}^{\infty} d x_{2} \int_{0}^{2 \pi / \omega_{0}}\left\{2 \rho \varepsilon \frac{\partial^{2} u_{i}}{\partial \theta \partial \tau} \cdot \hat{u}_{i}+\frac{1}{2} e_{i j k l m n} u_{k, l} u_{m, n} \hat{u}_{i, j}\right\} d \theta .
$$

We note that both terms in (2.65) are of order $\varepsilon^{2}$, and that in the above derivation we did not have to expand the equation of motion or the boundary condition.

With the use of (2.61) and (2.63), we obtain

$$
\begin{gathered}
u_{i, j}=\varepsilon \frac{\mathrm{i} \omega_{0}}{v} \sum_{m=-\infty}^{\infty} m f_{m}(\tau) Q_{i j}\left(x_{2}, m \omega_{0}\right) \mathrm{e}^{-\mathrm{i} m \omega_{0} \theta}, \\
\hat{u}_{i, j}=-\frac{\mathrm{i} k \omega_{0}}{v} \overline{Q_{i j}\left(x_{2}, k \omega_{0}\right)} \mathrm{e}^{\mathrm{i} k \omega_{0} \theta} .
\end{gathered}
$$

On substituting (2.66) and (2.67) into (2.65) and then evaluating the integral with respect to $x_{2}$, we obtain

$$
\frac{d f_{k}(\tau)}{d \tau}=\frac{\mathrm{i} k \omega_{0}^{2}}{4 \rho v^{2} N(1)} \sum_{m=-\infty}^{\infty} m(k-m) \mathcal{K}(k, m) f_{m} f_{k-m}, \quad k=1,2, \ldots,
$$

where $N(\omega)$ and $\mathcal{K}\left(\omega, \omega^{\prime}\right)$ are as in the previous subsection. As expected, by taking $\omega_{m}=m \omega_{0}$, identifying $f_{m}(\tau)$ with $\omega_{0} f\left(\omega_{m}, \eta\right)$, and then taking the limit $\omega_{0} \rightarrow 0$ in this subsection, all the sums tend to integrals and the evolution equations (2.68) recover the integral equation (2.57).

\section{(d) Evaluation of coefficients}

With the use of (2.61), the velocity on the surface $x_{2}=0$ is obtained as

$$
\frac{\partial \mathbf{u}}{\partial t}=\frac{\partial \mathbf{u}}{\partial \theta}=\varepsilon \sum_{n_{1}=1}^{\infty} g_{n_{1}}(\tau) \mathbf{d} \mathrm{e}^{-\mathrm{i} n_{1} \omega_{0} \theta}+C . C .+O\left(\varepsilon^{2}\right)
$$

where

$$
g_{n_{1}}(\tau)=-\mathrm{i} n_{1} \omega_{0} f_{n_{1}}(\tau) .
$$

In terms of $g_{n_{1}}$, the evolution equations (2.68) become

$$
\frac{d g_{n_{1}}(\tau)}{d \tau}=-\frac{n_{1}^{2} \omega_{0}}{4 \rho v^{2} N(1)} \sum_{n_{2}=-\infty}^{\infty} \mathcal{K}\left(n_{1}, n_{2}\right) g_{n_{2}} g_{n_{1}-n_{2}}, \quad n_{1}=1,2, \ldots
$$


16

It can easily be shown with the use of (2.60) that (2.71) can be rewritten as

$$
\begin{gathered}
\frac{d g_{n_{1}}(\tau)}{d \tau}=-\frac{n_{1}^{2} \omega_{0}}{4 \rho v^{2} N(1)}\left\{\sum_{n_{2}=1}^{n_{1}-1} \mathcal{K}\left(n_{1}, n_{2}\right) g_{n_{2}} g_{n_{1}-n_{2}}\right. \\
\left.-2 \sum_{n_{2}=n_{1}+1}^{\infty} \overline{\mathcal{K}\left(n_{2}, n_{1}\right)} g_{n_{2}} \overline{g_{n_{2}-n_{1}}}\right\}, \quad n_{1}=1,2, \cdots
\end{gathered}
$$

With the aid of the various expressions given in the previous subsection, we obtain for $n_{1}>n_{2}$,

$$
\mathcal{K}\left(n_{1}, n_{2}\right)=e_{i j k l m n} \sum_{r=1}^{3} \sum_{s=1}^{3} \sum_{t=1}^{3} \bar{A}_{i s} A_{k t} A_{m r} \frac{c_{r} \bar{c}_{s} c_{t} L_{n}^{(r)} \overline{L_{j}^{(s)}} L_{l}^{(t)}}{n_{2} p^{(t)}+\left(n_{1}-n_{2}\right) p^{(r)}-n_{1} \bar{p}^{(s)}}
$$

We have checked that the evolution equation (2.72) together with (2.73) is consistent with that given by Hamilton et al. (1999).

It is seen that to evaluate the coefficients in the evolution equations, we only need to know the values of $v, \mathbf{c}, p^{(1)}, p^{(2)}, p^{(3)}, A$. To find the linear surface-wave speed $v$ and the corresponding $M$, we may use the procedure stated in the paragraph below equation (2.15). Once $M$ is known, we may use (2.27) to find $E$ and hence $p^{(j)}, \mathbf{a}^{(j)}, j=1,2,3, A=\left[\mathbf{a}^{(1)}, \mathbf{a}^{(2)}, \mathbf{a}^{(3)}\right]$, and $\mathbf{c}=A^{-1} \mathbf{d}$.

The evolution equation (2.73) can be integrated numerically subjected to specified initial conditions. The evolution of surface velocity or surface elevation can then be determined. It is known that shocks always form at finite times. Parker and Talbot (1985) argued that the nonlocal character of the kernel in the evolution equation (2.73) might give rise to steady nonlinear surface waves and they calculated such solutions using a certain numerical procedure. Ogden and $\mathrm{Fu}$ (1996) questioned the validity of the procedure: they applied the same procedure to a simple example and obtained spurious solutions. Thus, the steady wave solutions obtained in Parker and Talbot (1985) were probably spurious as well.

It is not surprising that steady nonlinear surface waves cannot exist in the absence of any form of dispersion. When dispersion is introduced into the problem, it is expected that weak dispersion would balance the wave steepening effects of nonlinearity and lead to solitary wave solutions. In recent years, the existence of solitary wave solutions has been demonstrated for a variety of dispersion forms. Three major forms of dispersion are: (i) when the wavelength is large compared with the thickness of a coating layer, see Porubov and Samsonov (1995) and Eckl et al. (1998), (ii) when the material properties of the coating layer are close to those of the half-space, see Fu and Hill (2001), and (iii) when the half-space has a microstructure, see Porubov and Pastrone (2004) and Porubov et al. (2004). In Section 4, we shall illustrate the existence of solitary wave solutions by considering the first type of dispersion. 


\section{Linear wave propagation in a coated elastic half-space}

We now consider a coated half-space in which the half-space is made of the same material as in the previous sections but the coating layer has its constitutive relation given by

$$
\sigma_{i j}=\tilde{c}_{i j k l} u_{k, l}+\frac{1}{2} \tilde{e}_{i j k l m n} u_{k, l} u_{m, n}+\cdots,
$$

where $\tilde{c}_{i j k s}$ and $\tilde{e}_{i j k l m n}$ are tensors of first- and second-order elastic moduli. The coating layer is assumed to occupy the region $-h<x_{2}<0$. With the use of (2.8), we may deduce that such an elastic layer admits a travelling wave solution given by

$$
\begin{gathered}
\mathbf{u}=\left(\tilde{A}\left\langle\mathrm{e}^{\mathrm{i} \omega \tilde{p} x_{2} / v}\right\rangle \tilde{A}^{-1} \mathbf{d}^{(1)}+\overline{\tilde{A}}\left\langle\mathrm{e}^{\mathrm{i} \omega \tilde{\bar{p}} x_{2} / v}\right\rangle \overline{\tilde{A}}^{-1} \mathbf{d}^{(2)}\right) \mathrm{e}^{-\mathrm{i} \omega \theta} \\
=\left(\mathrm{e}^{-\tilde{E} k x_{2}} \mathbf{d}^{(1)}+\mathrm{e}^{\overline{\tilde{E}} k x_{2}} \mathbf{d}^{(2)}\right) \mathrm{e}^{-\mathrm{i} \omega \theta}
\end{gathered}
$$

where $k=\omega / v$ is the wavenumber, $\mathbf{d}^{(1)}$ and $\mathbf{d}^{(2)}$ are constant vectors to be determined, and here and hereafter a superimposed tilde signifies association with the elastic layer. Note that in (3.2) we have also included the solution corresponding to $\overline{\tilde{p}}_{1}, \overline{\tilde{p}}_{2}, \overline{\tilde{p}}_{3}$ since we do not require the solution to decay as $x_{2} \rightarrow \infty$. Corresponding to this solution, we have

$$
\begin{gathered}
\mathbf{u}(0)=\left(\mathbf{d}^{(1)}+\mathbf{d}^{(2)}\right) \mathrm{e}^{-\mathrm{i} \omega \theta} \\
\left.\left(\sigma_{i 2}\right)\right|_{x_{2}=0}=\mathrm{i} k\left(-\tilde{M} \mathbf{d}^{(1)}+\overline{\tilde{M}} \mathbf{d}^{(2)}\right) \mathrm{e}^{-\mathrm{i} \omega \theta}, \\
\left.\left(\sigma_{i 2}\right)\right|_{x_{2}=-h}=\mathrm{i} k\left(-\tilde{M} \mathrm{e}^{\tilde{E} k h} \mathbf{d}^{(1)}+\overline{\tilde{M}} \mathrm{e}^{-\tilde{E} k h} \mathbf{d}^{(2)}\right) \mathrm{e}^{-\mathrm{i} \omega \theta} .
\end{gathered}
$$

For the half-space, we have

$$
\begin{gathered}
\mathbf{u}=\mathrm{e}^{-E k x_{2}} \mathbf{d}^{(3)} \mathrm{e}^{-\mathrm{i} \omega \theta}, \\
\mathbf{u}(0)=\mathbf{d}^{(3)} \mathrm{e}^{-\mathrm{i} \omega \theta}, \\
\left.\left(\sigma_{i 2}\right)\right|_{x_{2}=0}=-\mathrm{i} k M \mathbf{d}^{(3)} \mathrm{e}^{-\mathrm{i} \omega \theta},
\end{gathered}
$$

where $\mathbf{d}^{(3)}$ is another constant vector to be determined. Thus, the traction-free boundary condition at $x_{2}=-h$ and the displacement and traction continuity at the interface $x_{2}=0$ give

$$
\begin{gathered}
-\tilde{M} \mathrm{e}^{\tilde{E} k h} \mathbf{d}^{(1)}+\overline{\tilde{M}} \mathrm{e}^{-\tilde{E} k h} \mathbf{d}^{(2)}=0, \\
\mathbf{d}^{(1)}+\mathbf{d}^{(2)}=\mathbf{d}^{(3)}, \\
-\tilde{M} \mathbf{d}^{(1)}+\tilde{\bar{M}} \mathbf{d}^{(2)}=-M \mathbf{d}^{(3)} .
\end{gathered}
$$

On eliminating $\mathbf{d}^{(1)}$ and $\mathbf{d}^{(2)}$ from the above equations, we obtain

$$
D \mathbf{d}^{(3)}=\mathbf{0}
$$


18

where

$$
D=\overline{\tilde{M}} \mathrm{e}^{-\overline{\tilde{E}} k h}-\left(\tilde{M} \mathrm{e}^{\tilde{E} k h}+\overline{\tilde{M}} \mathrm{e}^{-\bar{E} k h}\right)(\tilde{M}+\overline{\tilde{M}})^{-1}(M+\overline{\tilde{M}}) .
$$

Thus, the dispersion relation for the speed as a function of $k h$ is given by

$$
\operatorname{det} D=0 \text {. }
$$

For $k h \ll 1$, we obtain from $(3.13)$

$$
\begin{aligned}
& D=- M-k h\left\{\overline{\tilde{M} \tilde{E}}+(\tilde{M} \tilde{E}-\overline{\tilde{M}} \tilde{E})(\tilde{M}+\overline{\tilde{M}})^{-1}(M+\overline{\tilde{M}})\right\}+O\left(k^{2} h^{2}\right), \\
&=-M+k h\left(\tilde{R} \tilde{T}^{-1} \tilde{R}^{T}-\tilde{Q}+\tilde{\rho} v^{2} I-\mathrm{i} \tilde{R} \tilde{T}^{-1} M\right)+O\left(k^{2} h^{2}\right),
\end{aligned}
$$

where we have made use of the relation

$$
\tilde{M} \tilde{E}=\mathrm{i} \tilde{R} \tilde{T}^{-1} \tilde{M}-\tilde{R} \tilde{T}^{-1} \tilde{R}^{T}+\tilde{Q}-\rho v^{2} I
$$

which can be established with the aid of $(2.27)_{1}$ and (2.15).

Denote by $v_{R}$ the surface-wave speed associated with the half-space and by $\mathbf{d}$ the right eigenvector of $M$ when $v=v_{R}$ (so that $\mathbf{d}$ has the same meaning as in previous sections). We look for a solution of the form

$$
v=v_{R}+k h v_{1}+\cdots, \quad \mathbf{d}^{(3)}=\mathbf{d}+O(k h),
$$

where $v_{1}$ is a constant. We have

$$
M=\left.M\right|_{v=v_{R}}+\left.k h v_{1} \frac{d M}{d v}\right|_{v=v_{R}}+O\left(k^{2} h^{2}\right) .
$$

It is known (see, e.g., Mielke and Fu 2004) that

$$
\left.\frac{d M}{d v}\right|_{v=v_{R}}=-2 \rho v_{R} \int_{0}^{\infty} \mathrm{e}^{-x_{2} \bar{E}^{T}} \mathrm{e}^{-x_{2} E} d x_{2} .
$$

Thus,

$$
\left.\overline{\mathbf{d}} \cdot \frac{d M}{d v}\right|_{v=v_{R}} \mathbf{d}=-2 \rho v_{R} N\left(v_{R}\right)=-2 \rho N(1),
$$

where $N(1)$ is given by (2.58). On substituting (3.15)-(3.18) into $\overline{\mathbf{d}} \cdot D \mathbf{d}^{(3)}=0$ and equating the coefficients of $k h$, we obtain

$$
v_{1}=-\frac{\zeta}{2 \rho N(1)}, \quad \text { and so } v=v_{R}-\frac{\zeta k h}{2 \rho N(1)}+O\left(k^{2} h^{2}\right),
$$

where

$$
\zeta=\overline{\mathbf{d}} \cdot\left(\tilde{\rho} v_{R}^{2} I+\tilde{R} \tilde{T}^{-1} \tilde{R}^{T}-\tilde{Q}\right) \mathbf{d}
$$




\section{Solitary waves in a coated elastic half-space}

We now consider propagation of nonlinear travelling waves in the coated elastic half-space specified in the previous section. We shall consider a small-amplitude travelling wave solution in which the wavelength, $L$ say, is much greater than the plate thickness. To be more precise, we assume that $h / L$ is of the same order as $\varepsilon$ where $\varepsilon$ has the same meaning as in the previous sections. In order to keep track terms of different orders, we scale $x_{2}$ by $\varepsilon$, and still use $x_{2}$ for its scaled counterpart. The equation of motion $\sigma_{i j, j}=\tilde{\rho} \ddot{u}_{i}$, where $\tilde{\rho}$ is the material density of the layer, then becomes

$$
\begin{array}{r}
\frac{1}{\varepsilon^{2}} \tilde{T}_{i j} u_{j, 22}+\frac{1}{\varepsilon}\left(\tilde{R}_{i j}+\tilde{R}_{j i}\right) u_{j, 12}+\tilde{Q}_{i j} u_{j, 11}+\frac{1}{\varepsilon^{3}} \tilde{e}_{i 2 k 2 m 2} u_{k, 22} u_{m, 2} \\
+\frac{1}{\varepsilon^{2}}\left\{\tilde{e}_{i 2 k 2 m 1} u_{k, 22} u_{m, 1}+\left(\tilde{e}_{i 1 k 2 m 2}+\tilde{e}_{i 2 k 1 m 2}\right) u_{k, 12} u_{m, 2}\right\}+O(\varepsilon)=\tilde{\rho} \ddot{u}_{i},
\end{array}
$$

where

$$
\tilde{T}_{i k}=\tilde{c}_{i 2 k 2}, \quad \tilde{R}_{i k}=\tilde{c}_{i 1 k 2}, \quad \tilde{Q}_{i k}=\tilde{c}_{i 1 k 1} .
$$

A similar equation can be written down for the traction-free boundary condition $\sigma_{i 2}=0$ at $x_{2}=-h$. We now look for an asymptotic solution of the form

$$
\mathbf{u}=\varepsilon \mathbf{u}^{(1)}+\varepsilon^{2} \mathbf{u}^{(2)}+\varepsilon^{3} \mathbf{u}^{(3)}+O\left(\varepsilon^{4}\right) .
$$

On substituting (4.3) into (4.1), equating the coefficients of $\varepsilon^{-1}, \varepsilon^{0}$ and then solving the two equations subjected to the corresponding traction-free boundary conditions at $x_{2}=-h$, we find that $\mathbf{u}^{(1)}$ is independent of $x_{2}$ and that $\mathbf{u}^{(2)}$ is given by

$$
\mathbf{u}^{(2)}=-\tilde{T}^{-1} \tilde{R}^{T} \frac{\partial \mathbf{u}^{(1)}}{\partial x_{1}} x_{2}+\text { an arbitrary function independent of } x_{2} .
$$

With the aid of these results, we may evaluate the traction vector $\left(\sigma_{i 2}\right)$ at the interface $x_{2}=0$. It is found that its order $\varepsilon$ term is identically zero. To find the $O\left(\varepsilon^{2}\right)$ term, we could carry out the above expansion to the next order, but a simpler method is to rewrite the equation of motion as

$$
\sigma_{i 1,1}+\sigma_{i 2,2}=\tilde{\rho} \ddot{u}_{i}
$$

and then integrate across the layer thickness to obtain

$$
\left.\sigma_{i 2}\right|_{x_{2}=0}=\int_{-h}^{0}\left(\hat{\rho} \ddot{u}_{i}-\sigma_{i 1,1}\right) d x_{2} .
$$

With the use of (3.1) and (4.3), we obtain

$$
\left.\left(\sigma_{i 2}\right)\right|_{x_{2}=0}=\left.h\left\{\tilde{\rho} \ddot{\mathbf{u}}+\left(\tilde{R} \tilde{T}^{-1} \tilde{R}^{T}-\tilde{Q}\right) \mathbf{u}_{, 11}\right\}\right|_{x_{2}=0}+O\left(\varepsilon^{3}\right) .
$$

Because of continuity of displacement and traction across the interface, the $\mathbf{u}$ and $\left(\sigma_{i 2}\right)$ in (4.6) can be taken to their counterparts in the half-space. Equation (4.6) 
20

then becomes an effective boundary condition to be imposed on the surface of the half-space. Such an effective boundary condition was first given by Tiersten (1969) and later assumed in Eckl et al. (1998). We observe that this effective boundary condition does not contain nonlinear terms. Since the above asymptotic procedure is the same as that used by Porubov and Samsonov (1995), the latter authors have implicitly used such an effective boundary condition although they did not display this explicitly (they used the method of imposing solvability conditions to derive their evolution equations).

The matrix $\tilde{R} \tilde{T}^{-1} \tilde{R}^{T}-\tilde{Q}$ in (4.6) is recognized as the matrix $N_{3}$ that appears in the Stroh formulation. This is not surprising since the Stroh formulation gives

$$
\left(\sigma_{i 2}\right)_{, 2}=\tilde{\rho} \ddot{\boldsymbol{u}}+\left(\tilde{R} \tilde{T}^{-1} \tilde{R}^{T}-\tilde{Q}\right) \boldsymbol{u}_{, 11}-\tilde{R} \tilde{T}^{-1}\left(\sigma_{i 2}\right)_{, 1} .
$$

On integrating this equation from $-h$ to 0 and making use of the traction-free boundary condition at $x_{2}=-h$, we recover (4.6) in the limit $h \rightarrow 0$.

Since the leading order term in the effective boundary condition (4.6) is of order $\varepsilon^{2}$, the coated half-space behaves like an uncoated half-space to leading order and the leading-order solution (2.61) is still valid but we now expect that the effective boundary condition will affect the evolution of the amplitude functions $f_{m}(\tau)$. We have

$$
\begin{gathered}
\left.\left(\sigma_{i 2}\right)\right|_{x_{2}=0}=\left.h\left\{\tilde{\rho} v_{R}^{2} I+\tilde{R} \tilde{T}^{-1} \tilde{R}^{T}-\tilde{Q}\right\} \mathbf{u}_{, 11}\right|_{x_{2}=0}+O\left(\varepsilon^{3}\right) \\
=-\frac{\varepsilon h \omega_{0}^{2}}{v_{R}^{2}}\left(\tilde{\rho} v_{R}^{2} I+\tilde{R} \tilde{T}^{-1} \tilde{R}^{T}-\tilde{Q}\right) \mathbf{d} \sum_{m=1}^{\infty} m^{2} f_{m}(\tau) \mathrm{e}^{-\mathrm{i} m \omega_{0} \theta}+C . C .+O\left(\varepsilon^{3}\right) .
\end{gathered}
$$

The integral $I$ given by (2.62) is no longer zero; instead it is now given by

$$
I=-\left.\int_{0}^{2 \pi / \omega_{0}}\left(\sigma_{i 2} \hat{u}_{i}\right)\right|_{x_{2}=0} d \theta=\frac{\varepsilon h 2 \pi \omega_{0} \zeta}{v_{R}^{2}} k^{2} f_{k}(\tau),
$$

where $\zeta$ is given by $(3.21)$.

The evolution equation (2.68) is now replaced by

$$
\begin{gathered}
\frac{d f_{k}(\tau)}{d \tau}=\frac{h}{\varepsilon} \cdot \frac{\mathrm{i} \omega_{0}^{2} \zeta}{2 \rho v_{R}^{2} N(1)} k^{2} f_{k} \\
+\frac{\mathrm{i} k \omega_{0}^{2}}{4 \rho v_{R}^{2} N(1)} \sum_{m=-\infty}^{\infty} m(k-m) \mathcal{K}(k, m) f_{m} f_{k-m}, \quad k=1,2, \ldots,
\end{gathered}
$$

On neglecting the nonlinear term above, we find that the solution of (4.9) is given by

$$
f_{k}(\tau)=f_{k}(0) \exp \left\{k^{2} h \cdot \frac{\mathrm{i} \omega_{0}^{2} \zeta}{2 \rho v_{R}^{2} N(1)} t\right\} .
$$

On substituting this expression into (2.61), we find that the coating layer gives rise to a small correction to the wave speed and that the total wave speed is now given by

$$
v_{R}-\frac{\zeta}{2 \rho N(1)} \tilde{k} h+O\left(\tilde{k}^{2} h^{2}\right)
$$


where $\tilde{k}=k \omega_{0} / v$ is the actual wave number of the mode. This expression is consistent with the two-term expansion given by $(3.20)_{2}$.

The velocity at the interface $x_{2}=0$ is again given by (2.69). In terms of the $g_{n_{1}}(\tau)$ defined by $(2.70)$, the evolution equation (4.9) becomes

$$
\begin{aligned}
\frac{d g_{n_{1}}(\tau)}{d \tau}= & \frac{h}{\varepsilon} \cdot \frac{\mathrm{i} \omega_{0}^{2} \zeta}{2 \rho v_{R}^{2} N(1)} g_{n_{1}}-\frac{n_{1}^{2} \omega_{0}}{4 \rho v_{R}^{2} N(1)}\left\{\sum_{n_{2}=1}^{n_{1}-1} \mathcal{K}\left(n_{1}, n_{2}\right) g_{n_{2}} g_{n_{1}-n_{2}}\right. \\
& \left.-2 \sum_{n_{2}=n_{1}+1}^{\infty} \overline{\mathcal{K}\left(n_{2}, n_{1}\right)} g_{n_{2}} \overline{g_{n_{2}-n_{1}}}\right\}, \quad n_{1}=1,2, \cdots
\end{aligned}
$$

We may look for a solution of the form

$$
g_{n_{1}}(\tau)=\Gamma_{n_{1}} \mathrm{e}^{\mathrm{i} n_{1} c \tau}
$$

where $c$ is a real constant and $\Gamma_{n_{1}}, n_{1}=1,2, \ldots$ are complex constants to be determined. On substituting (4.12) into (4.11), we obtain

$$
\begin{aligned}
\mathrm{i} n_{1} c \Gamma_{n_{1}}= & \frac{h}{\varepsilon} \cdot \frac{\mathrm{i} \omega_{0}^{2} \zeta}{2 \rho v_{R}^{2} N(1)} \Gamma_{n_{1}}-\frac{n_{1}{ }^{2} \omega_{0}}{4 \rho v_{R}^{2} N(1)}\left\{\sum_{n_{2}=1}^{n_{1}-1} \mathcal{K}\left(n_{1}, n_{2}\right) \Gamma_{n_{2}} \Gamma_{n_{1}-n_{2}}\right. \\
& \left.-2 \sum_{n_{2}=n_{1}+1}^{\infty} \overline{\mathcal{K}\left(n_{2}, n_{1}\right)} \Gamma_{n_{2}} \overline{\Gamma_{n_{2}-n_{1}}}\right\}, \quad n_{1}=1,2, \cdots .
\end{aligned}
$$

This system of algebraic equation can be solved using the following procedure proposed by Parker and Talbot (1985). We first replace the infinite system by a finite sysmtem of $M$ equations. that is we assume that $\Gamma_{k}=0(k=M, M+1, \ldots)$. We start with $M=2$, in which case the two simultaneous equations can be solved exactly. Using each of these solutions as a starting solution, we increase $M$ in unit steps until a convergence criterion is satisfied. At each step, the finite system of quadratic equations can be solved using Mathematica. As suggested by Eckl and Mayer (1998), solitary wave solutions can be obtained in the limit $\omega_{0} \rightarrow \infty$.

Finally, we note that for a generally prestressed isotropic elastic half-space, smallamplitude perturbations are governed by the incremental equation of motion

$$
\chi_{i j, j}=\rho \ddot{u}_{i}
$$

and the incremental traction-free boundary condition takes the form

$$
\chi_{i 2}=0, \quad \text { on } x_{2}=0
$$

where the incremental stress tensor $\left(\chi_{i j}\right)$ is given by

$$
\chi_{i j}=\mathcal{A}_{j i l k}^{1} u_{k, l}+\frac{1}{2} \mathcal{A}_{j i l k n m}^{2} u_{k, l} u_{m, n}+\cdots,
$$


22

and expressions for the elastic moduli $\mathcal{A}_{j i l k}^{1}$ and $\mathcal{A}_{\text {jilknm }}^{2}$ in terms of the strain-energy function and the principal stretches can be found in Ogden (1984) or Appendix A of $\mathrm{Fu}$ and Ogden (1999). Thus, with $c_{i j k l}$ and $e_{i j k l m n}$ identified with $\mathcal{A}_{j i l k}^{1}$ and $\mathcal{A}_{\text {jilknm }}^{2}$, respectively, all the results obtained above are also valid for a generally prestressed isotropic elastic half-space or coated half-space. However, we remark that it would be too restrictive to assume that $\mathcal{A}_{\text {jilk }}^{1}$ satisfies the strong convexity condition. Instead, we assume that $\mathcal{A}_{j i l k}^{1}$ are such that the corresponding surface-impedance matrix is positive definite when $v=0$. This assumption is made so that the incremental dynamic problem is well-posed.

Acknowledgement: The author thanks Professor V.E. Gusev for reading an earlier version of these notes and for drawing his attention to the work of Professor V.P. Reutov.

\section{References}

1. Barnett, D.M. and Lothe, J. 1973 Synthesis of the sextic and the integral formalism for dislocations, Greens function and surface waves in anisotropic elastic solids, Phys. Norv. 7, 13-19.

2. Barnett, D.M. and Lothe, J. 1974 Consideration of the existence of surface wave (Rayleigh wave) solutions in anisotropic elastic crystals. J. Phys. F: Metal Physics 4, 671-686.

3. Barnett, D.M. and Lothe, J. 1985 Free surface (Rayleigh) waves in anisotropic elastic half-spaces: the surface impedance method. Proc. R. Soc. Lond. A402, 135-152.

4. Bigoni, D., Ortiz, M. and Needleman, A. 1997 Effect of interfacial compliance on bifurcation of a layer bonded to a substrate. Int. J. Solids Struct. 34, 43054326.

5. Biryukov, S.V. 1985 Impedance method in the theory of elastic surface waves. Sov. Phys. Acoust. 31, 350-354.

6. Cai, Z.X. and Fu, Y.B. 1999 On the imperfection sensitivity of a coated elastic half-space. Proc. R. Soc. Lond. A 455, 3285-3309.

7. Cai, Z.X. and Fu, Y.B. 2000 Exact and asymptotic stability analyses of a coated elastic half-space. Int. J. Solids Struct. 37, 3101-3119.

8. Chadwick, P. and Smith, G.D. 1977 Foundations of the theory of surface waves in anisotropic elastic materials. In Advances in Applied Mechanics (ed. C.-S. Yih), vol. 17, pp. 303-376. New York: Academic Press. 
9. David, E.A. 1985 A uniform asymptotic solution for nonlinear surface acoustic waves. Int. J. Eng. Sci. 23, 699-708.

10. Eckl, C., Mayer, A.P. and Kovalev, A.S. 1998 Do surface acoustic solitons exist? Physical Review Letters 81, 983-986.

11. Eckl, C., Kovalev, A.S. and Mayer, A.P. 2004 Solitary surface acoustic waves. Physical Review E 70, Art. No. 046604.

12. Farnell, G.W. 1970 Properties of elastic surface waves. In Physical Acoustics (ed. W.P. Mason and R.N. Thurston), 109-166. New York: Academic.

13. Fu, Y.B. 2005 An explicit expression for the surface-impedance matrix of a generally anisotropic incompressible elastic material in a state of plane strain. Int. J. Non-linear Mech. 40, 229-239.

14. Fu, Y.B. and Brookes, D.W. 2006 An explicit expression for the surfaceimpedance tensor of a compressible monoclinic material in a state of plane strain. IMA J. Appl. Maths 71, 434-445

15. Fu, Y.B. and Devenish, B. 1996 Effects of pre-stresses on the propagation of nonlinear surface waves in an incompressible elastic half-space. Q. Jl Mech. appl. Math. 49, 65-80.

16. Fu, Y.B. and Hill, S.L.B. 2001 Propagation of steady nonlinear waves in a coated elastic half-space. Wave Motion 34, 109-129.

17. Fu, Y.B. and Mielke, A. 2002 A new identity for the surface-impedance matrix and its application to the determination of surface-wave speeds. Proc. $R$. Soc. Lond. A458, 2523-2543.

18. Fu, Y.B. and Ogden, R.W. 1999 Nonlinear stability analysis of pre-stressed elastic bodies. Continuum Mech. Thermodyn. 11, 141-172.

19. Gusev, V.E., Lauriks, W. and Thoen, J. 1997 New theory for the time evolution of nonlinear Rayleigh waves in an isotropic solid. Physical Review B 55 (1997), 9344-9347.

20. Gusev, V.E., Lauriks, W. and Thoen, J. 1998 New evolution equations for the nonlinear surface acoustic waves on an elastic solid of general anisotropy. $J$. Acoust. Soc. Am. 103 (1998), 3203-3215.

21. Hamilton, M.F., Il'inskii, Yu.A., and Zabolotskaya, E.A. 1995 Evolution equations for nonlinear Rayleigh waves. J. Acoust. Soc. Am. 97, 891-897. 
22. Hamilton, M.F., Il'inskii, Yu.A., and Zabolotskaya, E.A. 1999 Nonlinear surface acoustic waves in crystals. J. Acoust. Soc. Am. 105, 639-651.

23. Harvey, A.P., Craine, R.E. and Syngellakis, S. 1992 Propagation of nonlinear surface acoustic-waves on elastic and piezoelectric solids. J. Mech. Phys. Solids 40, 1529-1542.

24. Harvey, A.P. and Tupholme, G.E. 1991 Nonlinear mode-coupling of 2 codirectional surface acoustic-waves on a piezoelectric solid. Int. J. Eng. Sci. 29, 987-998.

25. Harvey, A.P. and Tupholme, G.E. 1992 Propagation of anisotropic elastic and piezoelectric nonlinear surface acoustic-waves. Wave Motion 16, 125-135.

26. Hess, P. 2002 Surface acoustic waves in materials science. Physics Today 55, 42-47.

27. Hess, P. and Lomonosov, A.M. 2005 Nanotechnological applications of nonlinear surface acoustic waves: mechanism of brittle fracture. Appl. Surf. Sci. 248, 123-128.

28. Hess, P., Lomonosov, A.M. and Lucza, T. 2005 Nanotechnological applications of nonlinear surface acoustic waves: brittle fracture. J. De. Physique. IV JP125, 321-326.

29. Hunter, J.K. 1989 Nonlinear surface waves. Contemporary Math. 100, 185-202.

30. Ingebrigtsen, K.A. and Tonning, A. 1969 Elastic surface waves in crystals. Physical Review 184, 942-951.

31. Jerebtsov, S.N., Kolomenskii, Al. A., and Schuessler, H.A. 2004 Characterization of a polycrystalline material with laser-excited nonlinear surface acoustic wave pulses. Int. J. Thermophysics 25, 485-490.

32. Kalyanasundaram, N. 1981 Nonlinear surface acoustic waves on an isotropic solid. Int. J. Eng. Sci. 19, 279-286.

33. Kolomenskii, A. A., Lomonosov, A.M., Kuschnereit, R. and Hess, P. 1997 Laser generation and detection of strongly nonlinear elastic surface pulses. Physical Review Letter 79, 1325-1328.

34. Kolomenskii, A. A. and Schuessler, H.A. 2001 Nonlinear compression of giant surface acoustic wave pulses. Physics Letters A280, 157-161. 
35. Kolomenskii, Al. A., Lomonosov, A.M., Kuschnereit, R. and Hess, P. 2003 Nonlinear surface acoustic wave pulses in solids: laser excitation, propagation, interactions. Review of scientific instruments 74, 448-452.

36. Lardner, R.W. 1983 Nonlinear surface waves on an elastic solid. Int. J. Eng. Sci. 21, 1331-1342.

37. Lardner, R.W. 1984 Nonlinear Rayleigh-waves - harmonic-generation, parametric amplification, and thermoviscous damping. J. Appl. Phys. 55, 32513260.

38. Lardner, R.W. 1985 Waveform distortion and shock development in nonlinear Rayleigh-waves. Int. J. Eng. Sci. 23, 113-118.

39. Lardner, R.W. 1986 Nonlinear surface waves on an elastic solid of general anisotropy J. Elast. 16, 63-73.

40. Lardner, R.W. and Tupholme, G.E. 1986 Nonlinear surface waves on cubic materials J. Elast. 16, 251-265.

41. Maugin, G.A. 1999 Nonlinear waves in elastic crystals. Oxford University Press.

42. Mayer, A.P. 1995 Surface acoustic waves in nonlinear elastic media. Phys. Rep. 256, 237-366.

43. Mielke, A. and Sprenger, P. 1998 Quasiconvexity at the boundary and a simple variational formulation of Agmon's condition. J. Elast. 51, 23-41.

44. Mielke, A. and Fu, Y.B. 2004 Uniqueness of the surface-wave speed: A proof that is independent of the Stroh Formalism. Mathematics and Mechanics of Solids 9, 5-15.

45. Meegan, G.D., Hamilton, M.F., Il'inskii, Yu.A. and Zabolotskaya, E.A. 1999 Nonlinear Stoneley and Scholte waves. J. Acoust. Soc. Am. 106, 1712-1723.

46. Ogden, R.W. 1984 Non-linear Elastic Deformations. New York: Ellis Horwood.

47. Ogden, R.W. and Fu, Y.B. 1996 Nonlinear stability analysis of a pre-stressed elastic half-space. In Contemporary research in the mechanics and mathematics of materials (eds R.C. Batra and M.F. Beatty), CIMNE (Barcelona), 164-175.

48. Ogden, R.W. and Sotiropoulos, D.A. 1995 On interfacial waves in pre-stressed layered incompressible elastic solids. Proc. R. Soc. Lond. A 450, 319-341. 
49. Ogden, R.W. and Sotiropoulos, D.A. 1996 The effect of pre-stress on guided ultrasonic waves between a surface layer and a half-space. Ultrasonics 34, 491494.

50. Parker, D.F. 1988 Waveform evolution for nonlinear surface acoustic-waves. Int. J. Eng. Sci. 26, 59-75.

51. Parker, D.F. and Talbot, F.M. 1985 Analysis and computation for nonlinear Rayleigh waves of permanent form. J. Elast. 15, 389-426.

52. Parker, D.F., Mayer, A.P., and Maradudin, A.A. 1992 The projection method for nonlinear surface acoustic waves. Wave Motion 16, 151-162.

53. Parker, D.F. and Maugin, G.A. (eds) 1987. Recent Developments in Surface Acoustic Waves: Proceedings of European Mechanics Colloquium 226, Springer-Verlag Berlin.

54. Planat, M. 1985 Multiple scale analysis of the nonlinear surface acoustic wave propagation in anisotropic crystals. J. Appl. Phy. 57, 4911-4915.

55. Porubov, A.V. and Pastrone, F. 2004 Nonlinear bell-shaped and kink-shaped strain waves in microstructured solids. Int. J. Non-linear Mech. 39, 1289-1299.

56. Porubov A.V., Pastrone, F. and Maugin, G.A. 2004 Selection of two-dimensional nonlinear strain waves in micro-structured media. Comptes Rendus Mecanique 332, 513-518.

57. Porubov, A.V. and Samsonov, A.M. 1995 Long non-linear strain waves in layered elastic half-space. Int. J. Non-linear Mech. 30, 861-877.

58. Reutov, V.P. 1973 Use of the averaged variational principle for describing multiwave interactions of elastic surface waves. Radiophys. Quantum Electron. 16, 1307-1316.

59. Schuessler, H.A., Jerebtsov, S.N., and Kolomenskii, Al. A. 2003 Determination of linear and nonlinear elastic parameters from laser experiments with surface acoustic wave pulses. Spectrochimica Acta Part B - Atomic Spectroscopy 58, 1171-1175.

60. Shull, D.J., Hamilton, M.F., Il'insky, Yu. A. and Zabolotskaya, E.A. 1993 Harmonic generation in plane and cylindrical nonlinear Rayleigh waves. $J$. Acoust. Soc. Am. 94, 418-427.

61. Stroh, A.N. 1958 Dislocations and cracks in anisotropic elasticity. Phil. Mag. 3, 625-646. 
62. Stroh, A.N. 1962 Steady state problems in anisotropic elasticity. J. Math. Phys. 41, 77-103.

63. Tiersten, H.F. 1969 Elastic surface waves guided by thin films. J. Appl. Phys. 40, 770-789.

64. Tupholme, G.E. and Harvey, A.P. 1988 Nonlinear surface acoustic-waves on a piezoelectric solid. Int. J. Eng. Sci. 26, 1161-1168.

65. Tupholme, G.E. and Harvey, A.P. 1992 Intermodulation distortion of nonlinear piezoelectric surface waves. J. Mech. Phys. Solids 40, 1651-1661.

66. Zabolotskaya, E.A. 1992 Nonlinear propagation of plane and circular Rayleigh waves in isotropic solids. J. Acoust. Soc. Am. 91, 2569-2575. 